\title{
HEAT TRANSFER TO LAMINAR FLOW BETWEEN PARALLEL PLATES WITH INTERFACIAL HEAT GENERATION
}

\author{
SHIGERU MORI, TOSHIAKI INOUE AND AKIRA TANIMOTO \\ Department of Chemical Engineering, \\ Kanazawa University, Kanazawa 920
}

\begin{abstract}
Heat transfer to a fully developed laminar flow in a parallel-plate wall reactor, which is a variation of the so-called tube-wall reactor, was analyzed theoretically. The heat conduction in the reactor wall is accounted for, and it is assumed that an interfacial reaction simply causes uniform heat generation or such heat generation as decreases linearly along the flow.

The influence of the interfacial heat generation on the interfacial temperature was found to be significant, and the influence of the conductance ratio of wall to fluid $\boldsymbol{R}_{w}$ thereon was found to be more significant than for the case without heat generation. With respect to the local Nusselt number, when the heat generation varies along the direction of flow, the influence of the heat generation is significant and the influence of $\boldsymbol{R}_{w}$ tends to be increased.

In addition to the above, experiments were carried out in which heat was generated electrically at the interface. The experimental results agreed fairly well with the theoretical predictions.
\end{abstract}

\section{Introduction}

Several studies have been presented in recent years ${ }^{2,3,8}$ of a so-called open tubular reactor or tubewall reactor, which is employed for enzyme reactions or highly exothermic reactions. The merit of the tube-wall reactor in improved thermal sensitivity was pointed out not only in the theoretical work of Smith and Carberry ${ }^{8}$ but in the experimental study of Field et al., in which catalytic methanation using this reactor was examined to produce substitute natural gas ${ }^{2}$.

Analysis of the heat transfer in such a tube-wall reactor is very interesting from the viewpoint of temperature control of the reactor and of the effective removal of the heat generated by a reaction. However, analyses of heat transfer have been rather rare to date for heat generation or absorption at a fluidwall interface, caused by a chemical reaction or a phase change such as evaporation. When heat is generated or absorbed at the interface, it is expected that the interfacial temperature distribution is affected by the thermal resistance of the wall. Accordingly, it seems that an analysis accounting for thermal conduction in the wall is more significant in this case than in the case unaccompanied by an interfacial heat source.

In this study, a theoretical analysis was made of heat transfer to a laminar flow in a parallel-plate wall reactor, which is a variation of the tube-wall reactor, as a conjugated problem accounting for thermal Received August 20, 1977 . Correspondence concerning this article should
be addressed to S. Mori. T. Inoue is now with Sekisui Koji Co., Ltd., Osaka 530 conduction in the reactor wall. And the influence of interfacial heat generation and conduction in the wall were examined through the interfacial temperature and the local Nusselt number distributions. In addition, experiments in which the heat source was replaced by electric heating instead of chemical reaction at the interface were carried out to verify the applicability of the theoretical analysis.

\section{Theoretical Analysis}

Incompressible fluid, physical properties of which are invariant, flows between parallel plates $2 w$ in width under the condition of fully developed laminar flow. Heat generation occurs at both interfaces in the region of $x=0$ to $L$. The heat is transferred in this region to the ambient through a wall of thickness $\delta$, or to the fluid. The other parts of the surrounding walls are thermally insulated. The temperature at the outer surfaces of the walls of interest is uniformly specified, $t_{w}$. For $x \leqq 0$, the fluid is at a uniform temperature $t_{e}$. When it is assumed for the fluid that viscous dissipation and axial heat conduction are negligible, the dimensionless energy equation in steady state is given as

$$
3\left(1-y^{* 2}\right) \frac{\partial \Theta_{f}}{\partial \bar{x}}=\frac{\partial^{2} \Theta_{f}}{\partial y^{* 2}}
$$

with boundary conditions

$$
\begin{aligned}
& \bar{x}=0 ; \quad \Theta_{f}\left(0, y^{*}\right)=1 \\
& y^{*}=0 ; \quad \partial \Theta_{f} / \partial y^{*}=0 \\
& y^{*}=1, \bar{x}>0 ; \quad \Theta_{f}(\bar{x}, 1)=\Theta_{0}(\bar{x})
\end{aligned}
$$


where $y^{*}$ is a dimensionless distance from the flow axis. Equation (2) includes the unknown function $\Theta_{0}(\bar{x})$, which must be determined later.

The heat conduction equation is written as

$$
L^{* 2} \frac{\partial^{2} \Theta_{s}}{\partial x^{* 2}}+\frac{\partial^{2} \Theta_{s}}{\partial \bar{y}^{2}}=0
$$

with boundary conditions

$$
\begin{aligned}
& x^{*}=0 \text { and } x^{*}=1 ; \quad \partial \Theta_{s} / \partial x^{*}=0 \\
& \bar{y}=0 ; \quad \Theta_{s}=\Theta_{0}(\bar{x}) \\
& \bar{y}=1 ; \quad \Theta_{s}=0
\end{aligned}
$$

where $\bar{y}$ is a dimensionless distance from the fluid-wall interface in the direction perpendicular to the flow axis. Here it should be noticed that the dimensionless axial ordinate also is different from that for the fluid.

The conjugated boundary condition is given as

$$
-\left.R_{w} \frac{\partial \Theta_{s}}{\partial \bar{y}}\right|_{\bar{y}=0}=-\frac{\partial \Theta_{f}}{\left.\partial y^{*}\right|_{y^{*}=1}}+Q^{*}
$$

where $Q^{*}$ is the dimensionless interfacial heat generation, which is an arbitrarily specified function of $x$.

Now, it is assumed that the interfacial temperature is represented by the following infinite series, which includes unknown coefficient $\tau_{i}$.

$$
\Theta_{0}(\bar{x})=\sum_{i=0}^{\infty} \tau_{i} \bar{x}^{i}
$$

The solutions of Eqs. (1) and (3) are given by Eqs. (7) and (11), respectively ${ }^{5}$.

$$
\begin{aligned}
\Theta_{f}= & \sum_{i=0}^{\infty} \tau_{i} \bar{x}^{i}-\left(\tau_{0}-1\right) \sum_{m=1}^{\infty} C_{m} Y_{m}\left(y^{*}\right) e^{-8 \lambda m_{m}^{2} \bar{x} / 3} \\
& -\sum_{i=1}^{\infty} \tau_{i} \sum_{m=1}^{\infty} C_{m} Y_{m}\left(y^{*}\right) F_{i}(\bar{x})
\end{aligned}
$$

where

$$
\begin{array}{r}
F_{1}(\bar{x})=\left\{1-\exp \left(-8 \lambda_{m}{ }^{2} \bar{x} / 3\right)\right\} /\left(8 \lambda_{m}{ }^{2} / 3\right), \\
F_{i}(\bar{x})=i\left\{\bar{x}^{i-1}-F_{i-1}(\bar{x})\right\} /\left(8 \lambda_{m}{ }^{2} / 3\right) \\
(i=2,3,4, \cdots)
\end{array}
$$

Here, $\lambda_{m}$ and $Y_{m}\left(y^{*}\right)$ are the eigenvalue and eigenfunction, respectively, for the well-known Graetz problem for laminar flow between parallel plates. They should satisfy the following eigenvalue problem.

$$
\left.\begin{array}{c}
\frac{d^{2} Y_{m}}{d y^{* 2}}+\lambda_{m}^{2}\left(1-y^{* 2}\right) Y_{m}=0 \\
y^{*}=0 ; \quad d Y_{m} / d y^{*=-}=0 \\
y^{*}=1 ; \quad Y_{m}=0
\end{array}\right\}
$$

Expansion coefficient $C_{m}$ is represented by

$$
C_{m}=\frac{\int_{0}^{1}\left(1-y^{* 2}\right) Y_{m} d y^{*}}{\int_{0}^{1}\left(1-y^{* 2}\right) Y_{m}{ }^{2} d y^{*}}=\frac{\left.\frac{d Y_{m} \mid}{d y^{*} \mid}\right|_{y^{*}=1}}{\lambda_{m}{ }^{2} \int_{0}^{1}\left(1-y^{* 2}\right) Y_{m}{ }^{2} d y^{*}}
$$

$$
\begin{aligned}
\Theta_{s}= & (1-\bar{y}) \sum_{i=0}^{\infty} \frac{\tau_{i}}{i+1}+2 \sum_{m=1}^{\infty} \frac{\sinh \left\{m \pi L^{*}(1-\bar{y})\right\}}{\sinh \left(m \pi L^{*}\right)} \\
& \times \cos \left(m \pi x^{*}\right) \int_{0}^{1} \sum_{i=0}^{\infty} \tau_{i} \bar{x}^{i} \cos \left(m \pi x^{*}\right) d x^{*}
\end{aligned}
$$

To determine the unknown coefficient $\tau_{i}$, the procedure presented by Sell and Hudson ${ }^{6}$ is employed. From Eq. (5), we obtain

$$
\begin{aligned}
\left.R_{w} \int_{0}^{1} \frac{\partial \Theta_{s}}{\partial y}\right|_{i \grave{y}=0} \cos \left(j \pi x^{*}\right) d x^{*}=\left.\int_{0}^{1} \frac{\partial \Theta_{f}}{\partial y^{*}}\right|_{i y^{*}=1} \cos \left(j \pi x^{*}\right) d x^{*} \\
-\int_{0}^{1} Q^{*} \cos \left(j \pi x^{*}\right) d x^{*} \quad(j=0,1,2, \cdots)(12)
\end{aligned}
$$

Generally, $Q^{*}$ is an arbitrary function of $x^{*}$. However, in the case where the interfacial reaction is zero order, or in several cases similar to this, $Q^{*}$ becomes constant and independent of $x^{*}$. In such cases, the second term on the right-hand side of Eq. (12) is zero for $j \geqq 1$.

If $\tau_{i}$ is determined by the infinite set of simultaneous arithmetic equations Eq. (12), we will obtain the temperature profiles of the fluid as well as the wall and interfacial temperature distribution. The mixedmean temperature of the fluid and the local Nusselt number respectively are defined as follows:

$$
\begin{gathered}
\Theta_{m}=\int_{0}^{1}\left(1-y^{* 2}\right) \Theta_{f} d y^{*} / \int_{0}^{1}\left(1-y^{* 2}\right) d y^{*} \\
=\sum_{i=0}^{\infty} \tau_{i} \bar{x}^{i}+\frac{3}{2}\left(\tau_{0}-1\right) \sum_{m=1}^{\infty}\left\{C_{m} Y_{m}^{\prime}(1) / \lambda_{m}{ }^{2}\right\} e^{-8 \lambda_{m}^{2} \bar{x}^{2} / 3} \\
+\frac{3}{2} \sum_{i=1}^{\infty} \tau_{i} \sum_{m=1}^{\infty}\left\{C_{m} Y_{m}^{\prime}(1) / \lambda_{m}{ }^{2}\right\} F_{i}(\bar{x}) \\
N u=-\left.2 \frac{\partial \Theta_{f}}{\partial y^{*}}\right|_{y^{*}=1} /\left(\Theta_{m}-\Theta_{0}\right)
\end{gathered}
$$

where $Y_{m}^{\prime}(1)$ indicates the derivative of $Y_{m}\left(y^{*}\right)$ with respect to $y^{*}$ at $y^{*}=1$.

\section{Numerical Results and Discussion}

The first twenty-five eigenvalues and the eigenfunctions required to make theoretical calculations were determined by integrating Eq. (8) numerically according to the Runge-Kutta-Gill scheme. The expansion coefficients were evaluated by using Simpson's Rule. It was confirmed that the results obtained agreed very well with the previous data ${ }^{1,7}$.

In the theoretical calculation, $x^{*}$ was conveniently employed between two dimensionless ordinates, $x^{*}$ and $\bar{x}$. Accordingly, a dimensionless parameter $G z$ must be added instead of $\bar{x}$. Equation (6), which represents the interfacial temperature distribution, was approximated by the first six terms.

The dimensionless heat generation $Q^{*}$ is an arbitrarily specified function of $x^{*}$, as mentioned above. When the section of interest is short, however, $Q^{*}$ can be roughly approximated for most cases by the following linear function of $x^{*}$. 


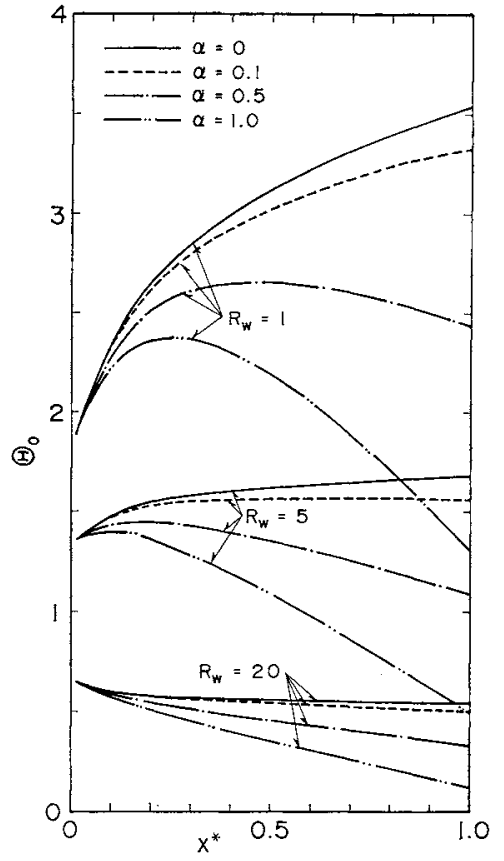

Fig. 1 Interfacial temperature distribution $\left(G z=100, L^{*}=0.01, Q_{0}=10\right)$

$$
Q^{*}=Q_{0}\left(1-\alpha x^{*}\right)
$$

When $\alpha=0, Q^{*}$ is constant and independent of $x^{*}$. This case represents the following two practical situations: first, where the interfacial reaction is of zero order, and second, where reactant concentration at the interface is uniform. For example, when the diffusion rate of the reactant in the fluid is much higher than that of the interfacial reaction, such a case can be approximated by the latter.

The distributions of both $\Theta_{0}$ and $N u$ were calculated for various values of parameters in the ranges $G z=1$ to $1000, R_{w}=1$ to 500 and $L^{*}=0.001$ to 0.1 . The ranges of the parameters with respect to the interfacial heat generation were selected as $Q_{0}=0$ to 50 and $\alpha=0$ to 1 . It was confirmed that the results obtained for $Q_{0}=0$ agreed with those of the previous work ${ }^{5}$.

Demonstrative numerical results are shown in Figs. 1 to 4 for $G z=100, L^{*}=0.01$ and $Q_{0}=10$. Figure 1 shows $\Theta_{0}$-distribution for several values of $R_{w}$. The influence of the heat generation is substantial. Furthermore, $\Theta_{0}$ decreases to a large degree with an increase in $R_{w}$ and the influence of heat transfer through the wall is very significant. The influence of $R_{w}$ is more important in the case with a heat source than in the case without $\mathrm{it}^{5)}$. When $R_{w}$ is small and $Q^{*}$ varies rapidiy along $x^{*}$, the distribution tends to show a maximum point clearly. This is very interesting because it means the appearance of a so-called hot spot, which is a serious problem in safe operation of a reactor. Although it is not shown, $\Theta_{0}$ is almost zero at $R_{v}=500$. Consequently, the influence of conduction in the wall is negligible for $R_{w} \geqq 500$ and

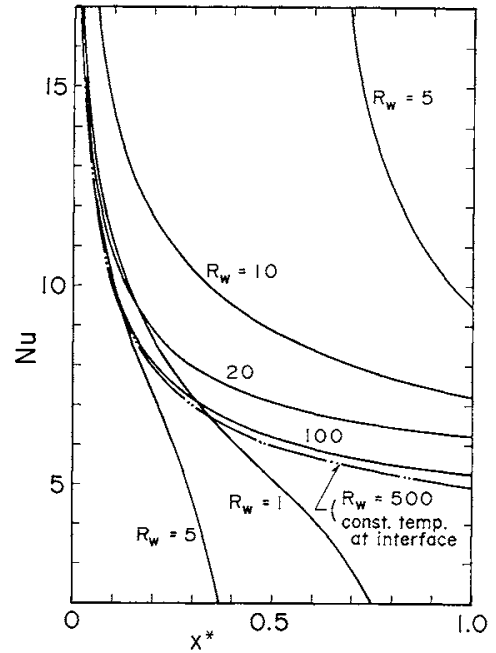

Fig. 2 Local Nusselt number $(G z=100$, $L^{*}=0.01, Q_{0}=10, \alpha=1$ )

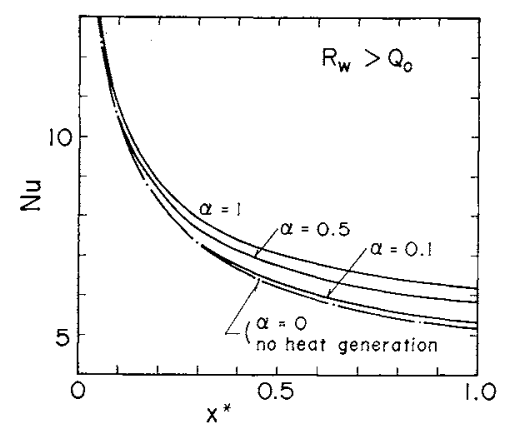

Fig. 3 Local Nusselt number for $\boldsymbol{R}_{w}>Q_{0}$ $\left(G z=100, L^{*}=0.01, Q_{0}=10, R_{w}=20\right)$

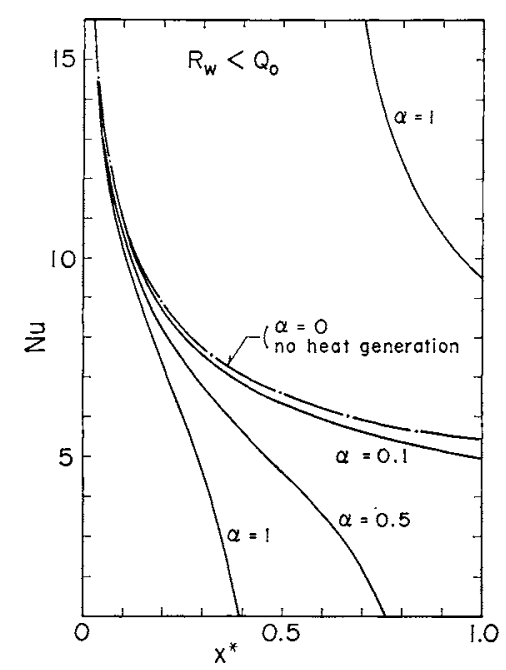

Fig. 4 Local Nusselt number for $\boldsymbol{R}_{w}<\boldsymbol{Q}_{0}$ $\left(G z=100, L^{*}=0.01, Q_{0}=10, R_{w}=5\right.$ )

this case can be treated as a uniform interfacial temperature case.

Regarding the influence of $G z$ on $\Theta_{0}$-distribution, it was found that the influence of heat generation was enlarged with decreasing $G z$. When $G z$ is small, it is expected that the heat generated at the interface is not 
easily transferred to the fluid and that it enforces an increase in $\Theta_{0}$. Therefore, the above result seems very reasonable. The effect of $L^{*}$ on $\Theta_{0}$ was so small that it could be neglected for practical purposes. The difference between results in the region of $L^{*}$, discussed in this paper, was $1 \%$ at most.

In Fig. 2, the influence of $R_{w}$ on $N u$-distribution is shown at $\alpha=1$. A discontinuity appears in the distribution for small $R_{w}$. Such discontinuity was also reported in several studies ${ }^{4,9}$ for heat-generating fluid. When $R_{w}$ is small, the heat generated at the interface is transferred to both the fluid and the wall, in the entrance region. In this region the fluid temperature rises along $x^{*}$. But on the contrary the heat generation rate decreases along $x^{*}$. Consequently, in the next region the interfacial temperature becomes lower than that of the fluid near the interface, and the heat flows inversely from the fluid to the wall. However, mixed-mean temperature is still lower than that at the interface. When both temperatures agree, there

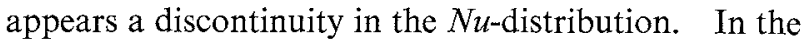
final region, the mixed-mean temperature becomes higher than that at the interface, and the Nusselt number becomes positive again.

When $R_{w}$ becomes much larger, $N u$-distribution approaches the Graetz solution of constant interfacial temperature, and finally almost agrees with it, at $R_{w}=500$. As a result, conduction in the wall can be neglected for $R_{w} \geqq 500$. This is the same as that derived from Fig. 1.

The influence of $G z$ was also examined. It was found that the influence of both interfacial heat generation and $R_{w}$ tended to be magnified with increasing Gz. Though such a tendency for the influence of $R_{w}$ is observed also in the case without a heat source at the interface, it is more significant in this case. But this conclusion is limited to the case where $Q^{*}$ is dependent on $x^{*}$. When $Q^{*}$ is constant and independent of $x^{*}$, the $N u$-distribution obtained agrees with that for $Q^{*}=0$. This is easily shown from Eqs. (12) and (14). In other words, uniform heat generation affects $\Theta_{0}$-distribution only, as expected.

Figures 3 and 4 show how $\alpha$ effects a change in $N u$-distribution, where $\alpha$ represents the dependence of $Q^{*}$ on $x^{*}$. From Fig. 3, for $R_{w}>Q_{0}$, it is seen that $\mathrm{Nu}$-distribution approaches that for the case without heat source as $\alpha$ approaches zero. Generally $N u$ tends to be higher with increasing $\alpha$ for the full range of $x^{*}$. On the other hand, a representative result for $R_{w}<Q_{0}$ is shown in Fig. 4. In this case, there is a tendency to show a discontinuity in $N u$-distribution. And the point at which the curve is discontinuous moves to the inlet with an increase in $\alpha$. For $R_{w}=Q_{0} N u$-distribution is independent of $\alpha$. This is assured from Eqs. (12) and (14). In short, when $\alpha$ approaches zero, $N u$-dis- tribution for $R_{v} \neq Q_{0}$ approaches that for the case without heat generation. In contrast with the above, $N u$-distribution for $R_{w}=Q_{0}$ is independent of $\alpha$ and different from the result for the case without heat source. Seemingly these two conclusions are inconsistent with each other. However, they are quite consistent because the type of the set of simultaneous arithmetic equations Eq. (14) changes according to whether $R_{w}=Q_{0}$ or not.

As a whole, the following conclusions were obtained from the above theoretical results. The influence of interfacial heat generation on $\Theta_{0}$-distribution is substantial. The influence of $R_{w}$ thereon is more significant than for the case without heat source. When $Q^{*}$ changes along $x^{*}$, the influence of $R_{w}$ on $\mathrm{Nu}$ distribution is enlarged and the heat generation affects $N u$-distribution significantly. The effect of $L^{*}$ is very small on both $\Theta_{0^{-}}$and $N u$-distributions and is usually negligible. In general, it can be concluded that conduction in the wall must be accounted for, especially when the heat is liberated at the interface.

\section{Experimental}

Experimental apparatus was similar on the whole to that in the previous work ${ }^{5}$ except for a portion in regard to heat generation at the interface. Air sent by a compressor flows into a duct at room temperature through a surge tank. Flow rate of the air was controlled by valves and an orifice flow-meter. The duct consists of three parts, the entrance section $2.2 \mathrm{~m}$, the test section $200 \mathrm{~mm}$ and the rear duct $200 \mathrm{~mm}$ in length, respectively. Cross-sectional shape of the duct is rectangular, $40 \mathrm{~mm}$ high (in the direction of $y^{*}$ ) and $120 \mathrm{~mm}$ wide (in the direction of $z$, which is perpendicular to $x-y$ plane). The test plates were mounted on both the upper and lower sides of the duct. The temperature at the outer surfaces of the test plates was maintained constant with jackets in which water was circulated through the constant temperature thermobox. The outside of the test section was sufficiently insulated thermally with foamed polystyrene. The test plates were made by joining polyvinyl chloride plates of suitable thickness by solvent. In the test plate, thermocouples $0.1 \mathrm{~mm}$ in diameter were installed in specified positions on the central axis of the duct. To generate heat at the interface, the surface of the test plate, which should be in contact with the fluid, was thinly grooved at $2 \mathrm{~mm}$ intervals in the direction perpendicular to the flow axis. Nichrome wire $0.2 \mathrm{~mm}$ in diameter was placed in grooves for electrical heating. The grooves were then filled with polyvinyl chloride dissolved in solvent, and the surface was smoothed. The nichrome heater had terminals at $10 \mathrm{~mm}$ intervals in the $x^{*}$-direction. The input voltage charged on each section of the heater 
was adjusted if required to the specified value. Thus, the distribution of heat generation at the interface could be arbitrarily changed.

In preparation, velocity profiles were measured by a hot-wire anemometer for various flow rates just in front of the test section and also behind it, and flow conditions were confirmed.

When the steady state was attained thermally after the apparatus had been set under the specified operating conditions, the temperature profiles of both the test plates and the fluid were measured. To measure the fluid temperature, a thermocouple $0.1 \mathrm{~mm}$ in diameter was inserted into the duct from its rear end. The thermocouple was tightly stretched, like a string on a bow, on the end of the guide. The guide was made of thin polyvinyl chloride plates so as to fit inside the duct. The operating conditions for the experiments are shown in Table 1. Physical properties of air were taken on an average of temperatures at the inlet and the outlet. The maximum change in local air temperature was about $13^{\circ} \mathrm{C}$ through all the experimental runs. This temperature change causes only about $5 \%$ deviation in air viscosity, which is the most sensitive of all the physical properties to temperature. Consequently, the physical properties can be approximated to be constant. The thermal conductivity of the polyvinyl chloride plate was taken to be $0.1325 \mathrm{~W} /(\mathrm{m} \cdot \mathrm{K})^{5}$.

From the results measured by the anemometer, it was shown that the velocity profile in the $y^{*}$-direction was parabolic and that the flow condition was a fully developed laminar flow in the central region in the $z$-direction of the duct, for the flow rates specified for the experiments. Such a velocity profile was almost invariant in a central region $90 \mathrm{~mm}$ in width.

The temperature profiles observed in the fluid and wall were in good agreement with the theoretical predictions. The interfacial temperature was estimated by the interpolation of temperature profiles in both the fluid and the wall. The temperature distributions at the interface obtained in this way are shown in Fig. 5 with the theoretical results. The experimental results of $\mathrm{Nu}$-distribution are given in Fig. 6. These experimental results were estimated according to the definition of Eq. (14).

All the above results shown in Figs. 5 and 6 taken together, it can be said that the theoretical results agree fairly well with the experimental data, though they differ somewhat from the experimentals in some runs.

\section{Conclusion}

Heat transfer to a fully developed laminar flow in a parallel-plate wall reactor was analyzed theoretically. In the analysis heat conduction in the wall was
Table 1 Operating conditions

\begin{tabular}{rrrrrrrrrr} 
Run & $t_{e}$ & $t_{w}$ & \multicolumn{1}{c}{$V$} & $\delta$ & \multicolumn{1}{c}{$G z$} & $R_{w}$ & $L^{*}$ & $Q_{0}$ & $\alpha$ \\
\hline 1 & 23.4 & 30.4 & 238 & 0.01 & 24.7 & 10.4 & 0.05 & -18.1 & - \\
2 & 18.7 & 31.1 & 666 & 0.01 & 69.2 & 10.4 & 0.05 & -10.5 & - \\
3 & 22.1 & 30.5 & 1080 & 0.01 & 112.2 & 10.4 & 0.05 & -15.4 & - \\
4 & 24.2 & 1.0 & 342 & 0.02 & 35.9 & 5.20 & 0.10 & 5.01 & 0.731 \\
5 & 22.4 & 0.9 & 893 & 0.02 & 92.7 & 5.20 & 0.10 & 5.43 & 0.730
\end{tabular}

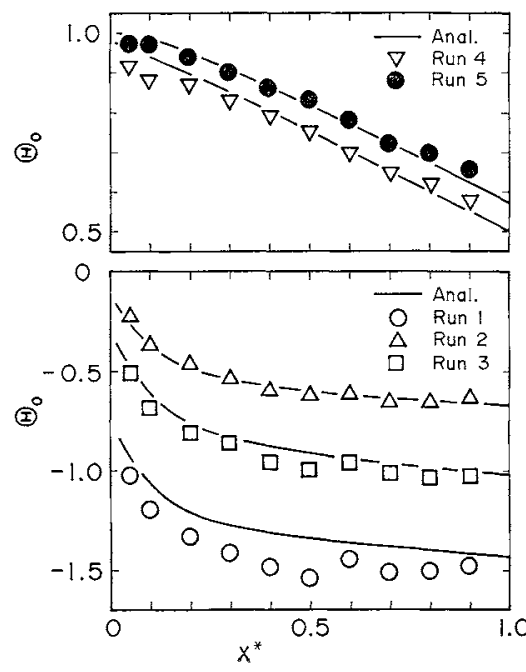

Fig. 5 Observed interfacial temperature

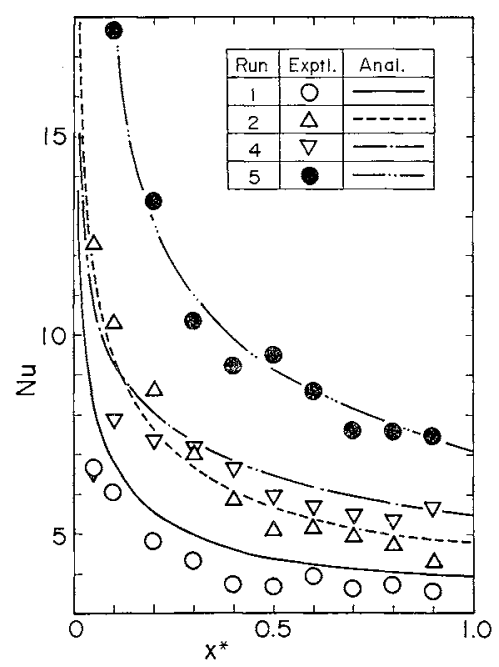

Fig. 6 Observed local Nusselt number

accounted for. Furthermore, experiments were carried out in which heat generation at the interface was replaced by electric heating. Interfacial heat generation was specified to be uniformly constant or a linear function with $x^{*}$ approximately, in both theory and experiment. The experimental results agreed fairly well with the theoretical predictions.

The influence of interfacial heat generation on interfacial temperature distribution is significant, and that of $R_{w}$ also is substantial. The influence of $R_{w}$ is more important than for the case without heat source. The factors which affect $\Theta_{0}$ are $G z, R_{w}, Q_{0}$ and $\alpha$. 
The influence of $L^{*}$ is so small that for practical purposes it can be neglected.

With respect to $N u$-distribution, when heat generation at the interface varies along $x^{*}$, the influence of heat generation is remarkable, and the influence of $R_{w}$ tends to be increased. There is no influence when heat generation is uniform, and $\mathrm{Nu}$-distribution agrees with that for the case without heat generation. The factors which affect $N u$ are $G z$ and $R_{w}$. And $Q_{0}$ and $\alpha$ are added to the above, except for the case where interfacial heat generation is uniformly constant. The influence of $L^{*}$ can be neglected on $\mathrm{Nu}$-distribution as well as on $\Theta_{0}$.

Taken all in all, it can be concluded that the influence of conduction in the wall is significant when accompanied by heat generation at the interface, compared to the case without heat generation.

\section{Acknowledgment}

The authors are grateful to Messrs. Shinju Tsukumo and Hiroshi Yamakawa for their assistance in the experimental work.

\section{Nomenclature}

$C_{m} \quad=$ coefficients in series expansion $\quad[-]$

$F_{i}() \quad=$ functions defined in Eq. (7) [-]

$G z \quad=$ Graetz number, $4 V W^{2} /(n L) \quad[-]$

$k \quad=$ thermal conductivity $\quad[\mathrm{W} /(\mathrm{m} \cdot \mathrm{K})]$

$L=$ length of heat-transporting section [m]

$L^{*} \quad=\delta / L \quad[-]$

$\mathrm{Nu} \quad=$ local Nusselt number based on width between parallel plates $\quad[-]$

$Q \quad=$ heat generation at fluid-wall interface $\left[\mathrm{W} / \mathrm{m}^{2}\right]$

$Q_{0} \quad=$ dimensionless heat generation at $x^{*}=0 \quad[-]$

$Q^{*} \quad=$ dimensionless heat generation at interface, $Q w /\left\{k_{f}\left(t_{e}-t_{w}\right)\right\}$

$R_{w} \quad=$ thermal conductance ratio, $k_{s} w /\left(k_{f} \delta\right)$

$t \quad=$ temperature

$V \quad=$ mean velocity of fluid

$w \quad=a$ half width between parallel plates

$x \quad=$ axial ordinate

\begin{tabular}{|c|c|c|}
\hline$x^{*}$ & $=x / L$ & {$[-]$} \\
\hline $\bar{x}$ & $=x_{\kappa} /\left(4 V w^{2}\right)$ & {$[-]$} \\
\hline$y$ & $=$ distance from flow axis & {$[\mathrm{m}]$} \\
\hline$y^{*}$ & $=y / w$ & {$[-]$} \\
\hline $\bar{y}$ & $=(y-w) / \delta$ & {$[-]$} \\
\hline$Y_{m}$ & $=$ eigenfunctions & {$[-]$} \\
\hline$\alpha$ & $=$ coefficient defined in Eq. (15) & {$[-]$} \\
\hline$\delta$ & $=$ thickness of wall & {$[\mathrm{m}]$} \\
\hline$\Theta$ & $=\left(t-t_{w}\right) /\left(t_{e}-t_{w}\right)$ & {$[-]$} \\
\hline$\kappa$ & $=$ thermal diffusivity of fluid & {$\left[\mathrm{m}^{2} / \mathrm{h}\right]$} \\
\hline$\lambda_{m}$ & $=$ eigenvalues & {$[-]$} \\
\hline$\tau_{i}$ & $=$ coefficients defined in Eq. (6) & {$[-]$} \\
\hline
\end{tabular}

$\langle$ Subscripts〉

$$
\begin{array}{ll}
e & =\text { entrance } \\
f & =\text { fluid } \\
m & =\text { mixed-mean } \\
s & =\text { solid wall } \\
w & =\text { outside surface of wall } \\
0 & =\text { fluid-wall interface }
\end{array}
$$

\section{Literature Cited}

1) Brown, G. M.: AIChE J., 6, 179 (1960).

2) Field, J. H., J. J. Demeter, A. J. Forney and D. Bienstock: Ind. Eng. Chem., Prod. Res. Dev., 3, 150 (1964).

3) Horvath, C. and B. A. Solomon: Biotech. Bioeng., 14, 885 (1972).

4) Mori, S., T. Inoue and A. Tanimoto: Can. J. Chem. Eng., 55, 138 (1977).

5) Mori, S., T. Shinke, M. Sakakibara and A. Tanimoto: Kagaku Kogaku Ronbunshu, 1, 235 (1975).

6) Sell, Jr., M. G. and J. L. Hudson: Int. J. Heat Mass Transfer, 9, 11 (1966).

7) Sellars, J. R., M. Tribus and J. S. Klein: Trans. ASME., 78, 441 (1956).

8) Smith, T. G. and J. J. Carberry: Chem. Eng. Sci., 30, 221 (1975).

9) Smorodinskii, E. L. and G. B. Froishteter: Teor. Osn. Khim. Tekhnol., 5, 542 (1971).

(Presented in part at the 14th Japan Heat Transfer Symposium at Tokyo, May, 1977.) 